\title{
Preliminary Report on the Flora of Southern Hidaka, Hokkaido (Yezo). XXVIII.
}

By

\section{Hiroshi Hara.}

Received January 18, 1938.

Brachypodium P. Beauvois.

$643^{(8) * B r a c h y p o d i u m ~ s y l v a t i c u m ~ P . ~ B e a u v o r s, ~ E s s . ~ A g r o s t . ~ p . ~ 101, ~ t . ~ 3, ~}$ f. 11 (1812).-Nevski in Fl. URSS. II, p. 594 (1934).

Syn. Festuca sylvatica Hudson, Fl. Angl. ed. 1, p. 38 (1762).

Festuca pinnata $\beta$. sylvatica Hudson, l. c. ed. 2, I, p. 48 (1778).

**var. villosum Lejeune et Courtois, Comp. Fl. Belg. I, p. 100(1828).

Syn. Bromus dumosus Villars, Hist. Pl. Dauph. II, p. 119 (1787).

Brachypodium sylvaticum a. spiculis villosis GaUdin, Fl. Helv. I, p. 305 (1828).

Brachypodium gracile var. pubescens Petermann, Fl. Lips. p. 70 (1838).

Brachypodium silvaticum $\beta$. dumosum Beck, Fl. N.-Öster. I, p. 110 (1890).

Brachypodium sylvaticum P. Beauvois, l. c.-Koidzumi, Fl. Symb. Or.-Asia. p. 80 (1930).-Mirabe et Kudo, Fl. Hokk. \& Saghal. II, p. 174 (1931).-Honda in Journ. Jap. Bot. XII, p. 153 (1936).

Brachypodium miserum (non KoIdzumi) Honda, Monogr. Poac. Jap. p. 32 (1930) pro parte.

Nom. Jap. Yezo-yamakamojigusa (KoIDzumi).

Hab. at the foot of Mt. Apoi. Fl. Jul.-early Aug.

*var. leiospicum HaRA, nom. nov.

Syn. Bromus gracilis (non Leysser 1761) Weigel, Obs. Bot. p. 15, t. 1, f. 11 (1772).

Brachypodium sylvaticum $\beta$. spiculis glaberrimis, scabris GAUdIN, l. c. (1828).

Brachypodium silvaticum a. typicum BECK, l. c. (1890).

Brachypodium miserum (non KoIdzumi) NakaI, Veg. Apoi p. 73 (1930).-Honda, l. c. (1930) pro parte--Miyabe et Kudo, l.c. p. 175 (1931).-Honda, l. c. p. 152 (1936) pro parte.

Brachypodium japonicum (non Miquel) Tatew., Veg. Apoi p. 117 (1928). 
Brachypodium sylvaticum var. miserum KoIDzumi, l. c. (1930) quoad descrip. et specim.

Folia vaginaque villosa. Glumae lemmaeque glabrae laeves vel leviter scabridae.

Nom. Jap. Ke-yamakamojigusa (nov.).

Hab. at the foot of Mt. Apoi, Samani, ete. Fl. Jul.

Dist. sp. Kyushu, Shikoku, Honshu, Yezo, Kuriles, Saghalien, Korea, China, Manchuria, Siberia, Tibet, Malaysia, India, Asia Minor, Caucasus, Europe and N. Airica.

Var. leiospicum is rather uncommon in Europe, although it is sometimes treated as var. typicum. B. sylvaticum var. miserrimum (HoNDA) seems to be a depauperate form of that variety. Dr. KoIDzumi referred specimens with villose leaves to his var. miserum which is based on Festuca misera Thunberg, but according to Thunberg's original description, it has glabrous leaves, and should be regarded as a separate variety which may be identical with var. luzoniense Hara (B. silvaticum subsp. luzoniense Inackel in Philip. Journ. Sci. I, suppl. p. 269 (1906).

Bromus L.

644 ${ }^{(9) *}$ Bromus remotiflorus Oriwi in Acta Phytotax. et Geobot. IV, p. 58 (1935).

Syn. Festuca pauciflorus Thunberg, Fl. Jap. p. 52 (1784).-Honda, Monogr. Poac. Jap. p. 55 (1930).-Masamune, Fl. \& Geobot. Stud. Yakusima p. 471 (1934).

Festuca remotiflora Steudel, Syn. Glum. I, p. 315 (1855).

Bromus pauciflorus (non Schumacher 1801) Hackel in Bull. Herb.

Boiss. VII, p. 713 (1899).-NakaI, Veg. Apoi p. 73 (1930).-Miyabe, et Kudo, Fl. Hokk. \& Saghal. II, p. 172 (1931).

Nom. Jap. Kitsune-gaya.

Hab. at the foot of Mt. Apoi, Nikanbetsu valley, etc. Fl. Jul.

Dist. Kyushu, Shikoku, Honshu, Yezo, Korea and China.

Brylkinia Fr. SchmidT.

645(10)*Brylkinia caudata Fr. Sсhмidt, Reis. Amur. u. Sachal. p. 199, t. VIII, f. 22-27 (1868).-NaKaI, Veg. Apoi p. 73 (1930).-Honda, Monogr. Poac. Jap. p. 89 (1930).-Miyabe et Kudo, Fl. Hokk. \& Saghal. II, p. 155 (1931).-Rochevitz in Fl. URSS. II, p. 354 (1934).

Syn. Alopecurus caudatus Thunberg in Nova Acta Ups. III, p. 208 (1780).

Ehrharta caudata Munro in A. Gray, Bot. Jap. p. 420 in adnota (1859).

Nom. Jap. Hogaeri-gaya. 
Hab. at the foot of Mt. Apoi. Fl. Jul.

Dist. Shikoku, Honshu, Yezo, S. Kuriles and Saghalien.

Calamagrostis Adanson.

646 ${ }^{(11)} *$ Calamagrostis epigeios Roтн, Tent. Fl. Germ. I, p. 34 (1788).Nakai, Veg. Apoi p. 73 (1930).-Honda, Monogr. Poac. Jap. p. 160 (1930).Miyabe et Kudo, Fl. Hokk. \& Saghal. II, p. 137 (1931).-Roshevitz in Fl. URSS. II, p. 194 (1934).-Hiтchсоск, Man. Grass. U. S. p. 319, f. 643 \& p. 820 (1935).-Oriwi in Acta Phytotax et Geobot. V, p. 228 \& 238 (1936). Syn. Arundo epigeios Linnaeus, Sp. Pl. ed. 1, I, p. 81 (1753).

Nom. Jap. Yama-awa.

Hab. at the foot of Mt. Apoi, Utabetsu, etc. Fl. Jul.-early Aug.

Dist. Formosa, Kyushu, Shikoku, Honshu, Yezo, S. Kuriles, Saghalien, Korea, China, Manchuria, Ussuri, Dahuria, Siberia, Caucasus, Europe and N. America.

$647^{(12)}$ *Calamagrostis hakonensis Francilet et Savatier, Enum. Pl. Jap. II, p. 168 (1876) nom. nud. \& p. 599 (1877).-NAKAI, Veg. Apoi p. 73(1930). -Honda, Monogr. Poac. Jap. p. 167 (1930).-Miyabe et Kudo, Fl. Hokk. \& Saghal. II, p. 141 (1931).-Masamune, Fl. \& Geobot. Stud. Yakusima p. 480 (1934).-OHwi in Acta Phytotax. et Geobot. V, p. 232 \& 240 (1936).

Nom. Jap. Hime-nogariyasu.

Hab. in grassy places of Mt. Apoi, Horoman valley, ete. Fl. Jul.mid. Aug.

Dist. Kyushu, Shikoku, Honshu, Yezo, S. Kuriles and China.

648 ${ }^{(13)}$ * Calamagrostis Langsdorfi Trinius, Gram. Unifl. Diss. p. 225, t. IV, f. 10 (1824) (ut Langsdorffi):-NAKAI in Bot. Mag. Tokyo XL, p. 488 (1926).-Hultén, Fl. Kamt. I, p. 100 (1927).-Tatew., Veg. Apoi p. 117 (1928).--NakaI, Veg. Apoi p. 74 (1930).-Honda, Monogr. Poac. Jap. p. 168 (1930).-Miyabe et Kudo, Fl. Hokk. \& Saghal. II, p. 139 (1931).-Komarov et Klob.-Alisova, Key. Pl. Far East. Reg. URSS. I, p. 186, t. 51 (1931).Roshevitz in Fl. URSS. II, p. 213 (1934).-Tatewaki et Kobayashi, Contr. Fl. Aleut. Is. p. 94 (1934).-Ohwi in Acta Phytotax. et Geobot. V, p. 229 $\& 238(1936)$.

Syn. Arundo Langsdorfi Link, Enum. Hort. Berol. I, p. 74 (1821).

Calamagrostis canadensis Michaux var. Langsdorfi InMAN in Rhodora 24, p. 143 (1922).-Stebbins in Rhodora 32, p. 43 (1930) quoad syn. Nom. Jap. Iwa-nogariyasu.

Hab. in grassy places of Mt. Apoi, Mt. Ruchishi, Mt. Rakko, etc. Fl. Jul. 
Dist. Shikoku, Honshu, Yezo, Kuriles, Saghalien, Korea, China, Manchuria, Amur, Dahuria, Kamtchatka, Siberia and Aleutian.

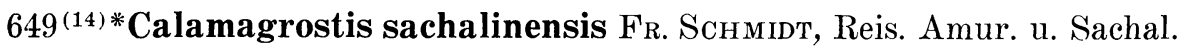
p. 202, t. VIII, f. 8-14 (1868).-TAtew., Veg. Apoi p. 117 (1928).-NAKaI, Veg. Apoi p. 74 (1930).-Honda, Monogr. Poac. Jap. p. 172 (1930).-Miyabe et Kudo, Fl. Hokk. \& Saghal. II, p. 141 (1931).-Roshevitz in Fl. URSS. II, p. 221 (1934).-Ohwi in Acta Phytotax. et Geobot. V, p. 231 \& 239 (1936).

Nom. Jap. Takane-nogariyasu (Matsumura).

Hab. in grassy slopes on Mt. Apoi, Mt. Horoman, Mt. Toyoni, Mt. Rakko, ete. Fl. Jul.-mid. Aug.

Dist. Shikoku, Honshu, Yezo, Kuriles, Saghalien and Kamtchatka.

Deschampsia P. Beauvors.

$650^{(15)}$ Deschampsia caespitosa P. Beauvors, Ess. Agrost. p. 91, t. 18, f. 3 (1812).-Honda, Monogr. Poac. Jap. p. 138.(1930).-Miyabe et Kudo, Fl. Hokk. \& Saghal II, p. 146 (1931).-Tatewaki \& Kobayasin, Contr. Fl. Aleut. Is. p. 97 (1934).-Masamune, Fl. \& Geobot. Stud. Yakusima p. 477 (1934).-Hiтchсоск, Man. Grass. U. S. p. 289, f. 576 \& p. 833 (1935).

Syn. Aira caespitosa Linnaeus, Sp. Pl. ed. 1, I, p. 64 (1753).

subsp. orientalis Hultén, Fl. Kamt. I, p. 109, pl. 5, fig. a, b (1927).

Syn. Deschampsia borealis Roshevitz in Fl. URSS. II, p. 246 \& 750 (1934) saltem pro parte.

Nom. Jap. Yezo-miyama-komesusuki (nov.).

Hab. at C. Erimo. Fl. Aug.

Dist. sp. Formosa, Kyushu, Shikoku, Honshu, Yezo, Kuriles, Saghalien, Korea, China, Manchuria, Amur, Dahuria, Kamtchatka, Siberia, Himalaya, Caucasus, Europe and N. America.

Our specimen has big spikelets about $5 \mathrm{~mm}$. long. Glumes are 3.5$4.5 \mathrm{~mm}$. long.

Diarrhena P. Beauvors (nom. conserv.).

651 (16)*Diarrhena japonica Franchet et Savatier, Enum. Pl. Jap. II, p. 603 (1877).--TAtew., Veg. Apoi p. 117 (1928).-NakaI, Veg. Apoi p. 74 (1930).-Honda, Monogr. Poac. Jap. p. 94 (1930).-Miyabe et Kudo, Fl. Hokk. \& Saghal. II, p. 154 (1931).

Syn. Onœa japonica Franchet et Savatier, l. c. p. 178 (1876).

Nom. Jap. Tatsunohige.

Hab. in forests at the foot of Mt. Apoi. Fl. Jul.

Dist. Kyushu, Shikoku, Honshu and Yezo. 


\section{Digitaria HeIster.}

652(17) Digitaria ischaemum Muenlenberg, Deser. Gram. Amer. p. 131 (1817).-Нiтснсоск, Man. Grass. U. S. p. 557, f. 1162 \& p. 836 (1935).

Syn. Panicum lineare (non L. 1762) Krocker, Fl. Siles. I, p. 95 (1787).

Panicum ischaemum Schreber ex Schweigger, Spec. Fl. Erlang. p. 16 (1804).

Digitaria linearis (non Persoon 1805) Crepin, Man. Fl. Belg. ed. 2, p. 335 (1866).-Roshevitz in Fl. URSS. II, p. 28 (1934).

Syntherisma Ischaemum NASH in N. Amer. Fl. 17-2, p. 151 (1912).-

Honda, Monogr. Poac. Jap. p. 292 (1930).-Miyabe et Kudo, Fl. Hokk. \& Saghal. II, p. 115 (1931).-Masamune, Fl. \& Geobot. Stud. Yakusima p. 493 (1934).

Nom. Jap. Aki-mehijiwa (Makino).

Hab. near Saruru. Fl. Aug.

Dist. Formosa, Liukiu, Kyushu, Shikoku, Honsyu, Yezo, Saghalien, Korea, China, Ussuri, Amur, Dahuria, Siberia, Cancasus and Europe. Naturalized in America.

\section{Echinochloa P. Beauvors.}

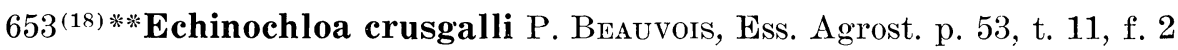
(1812).-Honda in Bot. Mag. Tokyo XXXVII, p. 119 (1923); Monogr. Poac. Jap. p. 263 (1930).-Miyabe et Kudo, Fl. Hokk. \& Saghal. II, p. 116 (1931.).-Roshevitz in Fl. URSS. II, p. 32 (1934).-Hiтchсоск, Man. Grass. U. S. p. 692 , f. 1553 \& p. 839 (1935).

Syn. Panicum Crusgalli Linnaeus, Sp. Pl. ed. 1, I, p. 56 (1753). subsp. genuina Honda, l. c. p. 120 (1923); l. c. (1930). var. longiseta (Trinius) Hara, comb. nov.

Syn. Panicum crusgalli var. longiseta Trinius, Sp. Gram. Icon. II, t. 162 (1829) in textu.

Panicum crusgalli var. longisetum DöLL, Fl. Bad. I, p. 232 (1857).

Echinochloa crusgalli subsp. genuina var. echinata Honda, 1. c. p. 120

(1923) ; l. c. p. 264 (1930).-Miyabe et Kudo, l. c. p. 117 (1931).

Nom. Jap. Midzu-bie, Kuma-bie.

Hab. at the foot of Mt. Apoi. Fl. Aug.

Dist. sp. Formosa, Liukiu, Kyushu, Shikoku, Honshu, Yezo, Saghalien, Korea, China, Manchuria, Ussuri, Amur, Siberia, India, Caucasus, Europe, Africa, America and Australia.

Elymus L.

654 ${ }^{(19)}$ *Elymus dahuricus Turczaninow [Catal. Pl. Baic.-Dah. no. 1331 (1838) nom. nud.] in Bull. Soc. Imp. Nat. Moscou XXIX-1, p. 61 (1856).- 
Grisebacin in Ledebour, Fl. Ross. IV, p. 331 (1853).--Nakai, Veg. Apoi p. 74 (1930).-Honda, Monogr. Poac. Jap. p. 18 (1930).-Miyabe et Kudo, Fl. Hokk. \& Saghal. II, p. 179 (1931).

Syn. Clinelymus dahuricus Nevski in Bull. Jard. Bot. Acad. Sci. URSS. XXX, p. 645 (1932) ; in Fl. URSS. II, p. 691 (1934).

Nom. Jap. Hama-mugi.

Hab. in coastal places of Mt. Apoi. Fl. Aug.

Dist. Kyushu (Tsushima), Honshu, Yezo, S. Kuriles, Saghalien, Korea, N. China, Manchuria, Ussuri, Dahuria, Mongolia, Siberia and Turkestan.

Two forms are found in this district. One is green in all parts (f. viridis), and the other entirely glaucous. (f. glaucus).

655(20) Elymus mollis Trinius in Sprengel, Neue Entd. II, p. 72 (1821).Honda, Monogr. Poac. Jap. p. 20 (1930).-Miyabe et Kudo, Fl. Hokk. \& Saghal. II, p. 177 (1931).-Komarov et Klob.-Alısova, Key Pl. Far East. Reg. USSR. I, p. 241, t. 66 (1931).-Nevski in Fl. URSS. II, p. 695 (1934).Tatewaki et Kobayasini, Contr. Fl. Aleut. Is. p. 98 (1934).-Hitcircock, Man. Grass. U. S. p. 249 f. 479, \& p. 845 (1935).

Syn. Elymus arenarius L. subsp. mollis Hultén, Fl. Kamt. I, p. 153(1927).

Leymus mollis (Trinius) Hara, mss.

Nom. Jap. IIama-ninniku.

Hab. on sandy coasts of C. Erimo, and between Shoya \& Saruru. Fl. Jul.

Dist. Honshu, Yezo, Kuriles, Saghalien, Korea, Ussuri, Amur, Ochotsk, Kamtchatka, Anadyr, Aleutian, N. America and Greenland.

\section{Festuca L.}

$656^{(21) * F e s t u c a}$ ovina Linnaeus, Sp. Pl. ed. 1, I, p. 73 (1753).-Tatew., Veg. Apoi p. 117 (1928).-Nakai, Veg. Apoi p. 74 (1930).-Honda, Monogr. Poac. Jap. p. 47 (1930).-Miyabe et Kudo, Fl. Hokk. \& Saghal. II, p. 168 (1931).-Masamune, Fl. \& Geobot. Stud. Yakusima p. 471 (1934).--HitchCock, Man. Grass. U. S. p. 76, f. 101 \& p. 860 (1935).

Nom. Jap. Ushinokegusa.

Hab. on Mt. Apoi and Mt. Horoman. Fl. Jul.

Dist. Formosa, Kyushu, Shikoku, Honshu, Yezo, Kuriles, Saghalien, Korea, China, Manchuria, Ussuri, Amur, Dahuria, Kamtchatka, Siberia, Caucasus, Europe and N. America.

This and the next species are treated here in a wide sense.

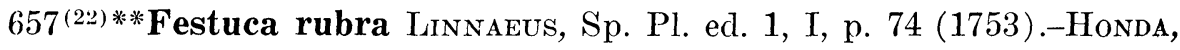


Monogr. Poac. Jap. p. 51 (1930).-Miyabe et Kudo, Fl. Hokk. \& Saghal. II, p. 169 (1931).-НІтснсоск, Man. Grass. U. S. p. 75 f. $97 \&$ p. 861 (1935).

Nom. Jap. Ô-ushinokegusa.

Hab. at the foot of Mt. Apoi, C. Erimo, ete. Fl. Jul.

Dist. Honshu, Yezo, Kuriles, Saghalien, Korea, China, Manchuria, Amur, Dahuria, Siberia, Himalaya, Caucasus, Europe, Africa and N. America.

Glyceria R. BRown (nom. conserv.).

$658^{(23)}$ Glyceria alnasteretum Komarov in Fedde, Rep. Sp. Nov. XIII, p. 87 (1914) ; Fl. Penin. Kamt. I, p. 182 (1927).-Hultén, Fl. Kamt. I, p. 138 f. 11 (1927).-Miyabe et Kudo, Fl. Hokk. \& Saghal. II, p. 166 (1931).-Oriwi in Acta Phytotax. et Geobot. II, p. 280 (1933).-Komarov, Fl. URSS, II, p. 457 (1934).-Handel-Mazzetri, Symb. Sin. VII-5, p. 1285 (1936) in nota. Syn. Glyceria remota var. japonica IIAckel in Bull. Herb. Boiss. VII, p. 712 (1899).

Glyceria arundinacea (non Kunth) Honda, Monogr. Poac. Jap. p. 63 (1930).-Miyabe et Kudo, l. c. (1931).

Nom. Jap. Miyama-dojôtsunagi.

Hab. along Panke R. Fl. Jul.

Dist. Honshu, Yezo, Kuriles and Kamtchatka.

$659^{(24) * G l y c e r i a ~ l e p t o l e p i s ~ O H w ~ i n ~ B o t . ~ M a g . ~ T o k y o ~ X L V, ~ p . ~} 381$ (1931) excl. syn. Hultén.

Syn. Glyceria aquatica (non Wahlenberg) Tatew., Veg. Apoi p. 117 (1928).-Nakai, Veg. Apoi p. 75 (1930).-Honda, Monogr. Poac. Jap. p. 62 (1930).-Miyabe et Kudo, Fl. Hokk. \& Saghal. II, p. 165 (1931).

?Glyceria ussuriensis Komarov, Fl. URSS. II, p. 460 \& 758 (1934).

Nom. Jap. Hirohano-dojôtsunagi.

Hab. in humid places at the foot of Mt. Apoi. Fl. Aug.

Dist. Kyushu, Honshu, Yezo, Saghalien, Korea and Ussuri?

Hierochloë R. Brown (nom. conserv.).

$660^{(25) * H i e r o c h l o e ̈ ~ o d o r a t a ~ P . ~ B e a u v o r s, ~ E s s . ~ A g r o s t . ~ p . ~ 164, ~ p l . ~ 12, ~ f . ~} 5$ (1812) (ut Hierochloa).-Hiтciсоск, Man. Grass. U. S. p. 527 f. 1111 \& p. 869 (1935).

Syn. Holcus odoratus Linnaeus, Sp. Pl. ed. 1, II, p. 1048 (1753).

*var. pubescens Krylov, Fl. Alt. p. 1553 (1914).

Syn. Hierochloe Bungeana Trinius in Mém. Acad. Sci. St.-Pétersb. ser. 6, V, p. $82(1839-40)$.-Roshevitz in Fl. URSS. II, p. 62 (1934).- 
Miyabe et Tatewaki in Trans. Sapporo Nat. Hist. Soc. XV, p. 46 (1937).

Hierochloe odorata var. sachalinensis PRINTz in Kgl. Norsk. Vidensk. Selsk. Skr. 1916-3, p. 8, t. 1 (1917).

Hierochloe odorata (non P. Beauvois) Tatew., Veg. Apoi p. 116 (1928).-Honda, Monogr. Poac. Jap. p. 220 (1930) pro major. part. -Miyabe et Kudo, Fl. Hokk. \& Saghal. II, p. 126 (1931).

Hierochloe odorata var. Trautvetteriana V. Petrov, Fl. Jakut. I, p. 130, f. 39 (1930).

Hierochloe odorata subsp. pubescens (KRYLOV) HARA, mss.

Nom. Jap. Kôbô.

Hab. in grassy places at the foot of Mt. Apoi, Samani, C. Erimo, etc. Fl. May-Jun.

Dist. Kyushu, Honshu, Yezo, Kuriles, Saghalien, Korea, China, Ussuri, Amur, Mongolia, Kamtchatka and Siberia.

Japanese specimens have generally retrorsely pubescent sheeths and glabrescent lemmas. The distinction between this form and the typical one, however, is not clear as HuLtén had already pointed out in his Fl. Kamt. I, p. 86. In Honshu, forms with glabrous sheaths or hairy lemmas or with sheaths annularly hairy only at the top (var. annulata V. PETrov) are also sometimes found. The specimen of the district has often low stems about $10 \mathrm{~cm}$. high. Its spiklets are large (about $5 \mathrm{~mm}$. long). Its leaves often hairy on the upper surface. Though I don't see the original description of $H$. Bungeana, it is strange to me that STEUder describes that species as having glabrous sheaths.

\section{Hystrix MoEnch.}

661 ${ }^{(26) * * H y s t r i x ~ l o n g e-a r i s t a t a ~ H o n d a, ~ M o n o g r . ~ P o a c . ~ J a p . ~ p . ~} 14$ (1930). Syn. Asprella sibirica var. longe-aristata Hackel in Bull. Herb. Boiss. ser. 2, IV, p. 525 (1904).

Nom. Jap. Adzuma-gaya.

Hab. in shady places at the Horoman side of Mt. Apoi. Fl. Jul.

Dist. Kyushu, Honshu, Yezo and Korea. 